\title{
Three-dimensional computed tomography evaluation of craniofacial characteristics according to lateral deviation of chin
}

\author{
Hyo-Won CHOI, Bola KIM, Jae-Young KIM, Jong-Ki HUH and Kwang-Ho PARK (D)
}

\begin{abstract}
Background: The relationship between the lateral deviation of chin and the upper and middle facial third asymmetry is still controversial. The purpose of this study is to evaluate the correlation of upper and middle facial third asymmetry with lateral deviation of chin using 3-dimensional computed tomography. The study was conducted on patients who underwent orthognathic surgery from January 2016 to August 2017. A total of 40 patients were included in this retrospective study. A spiral scanner was used to obtain the 3-dimensional computed tomography scans. The landmarks were assigned on the reconstructed 3-dimensional images, and their locations were verified on the axial, midsagittal, and coronal slices. The Pearson correlation analysis was performed to evaluate the correlation between chin deviation and difference between the measurements of distances in paired craniofacial structures. Statistical analysis was performed at a significance level of 5\%.

Results: In mandible, the degree of chin deviation was correlated with the mandibular length and mandibular body length. Mandibular length and mandibular body length are shorter on the deviated-chin side compared to that on the non-deviated side (mandibular length, $r=-0.897, p$ value $<0.001$; mandibular body length, $r=-0.318$, $p$ value $=0.045$ ). In the upper and middle facial thirds, the degree of chin deviation was correlated with the vertical asymmetry of the glenoid fossa and zygonion. Glenoid fossa and zygonion are superior on the deviated-chin side than on the non-deviated side (glenoid fossa, $r=0.317$, $p$ value $=0.046$; zygonion, $r=0.357, p$ value $=0.024$ ).

Conclusion: Lateral deviation of chin is correlated with upper and middle facial third asymmetry as well as lower facial third asymmetry. As a result, treatment planning in patients with chin deviation should involve a careful evaluation of the asymmetry of the upper and middle facial thirds to ensure complete patient satisfaction.
\end{abstract}

Keywords: Lateral deviation of chin, Upper and middle facial third asymmetry, Asymmetry of glenoid cavity

\section{Background}

Facial asymmetry is a relatively common feature with a prevalence rate of $21-85 \%$. In majority of cases, facial asymmetry is mild and hardly recognizable, and hence, surgical intervention is not usually necessary [1-4]. However, patients with apparent facial asymmetry may not be satisfied with their appearance; such patients are more likely to opt for surgical intervention for esthetic and occlusal improvement [3].

The most common type of facial asymmetry is observed in the lower third of face with lateral deviation of

\footnotetext{
* Correspondence: omspark@yuhs.ac

Department of Oral and Maxillofacial Surgery, Gangnam Severance Hospital, Yonsei University College of Dentistry, Seoul, Korea
}

the chin (75\%) [3]. The most common cause is unilateral mandibular hyperplasia, i.e., enlargement of the mandible [5]. Functional disharmony of the masticatory muscles may be associated with lower facial third asymmetry with lateral deviation of chin [6].

Facial asymmetry often involves varying degrees of upper (5\%) and middle (36\%) facial third asymmetries [3]. In a previous study, asymmetry of the glenoid cavity, a type of upper and middle facial third asymmetry, was reported [7]. Asymmetry of the glenoid cavity is caused by defects in generation, proliferation, migration, and differentiation of cranial neural crest cells [8] or craniofacial structure modeling from the cerebrum [9-11]. As a result, asymmetry of the glenoid cavity causes lateral deviation of the chin [7]. 
The relationship between the lateral deviation of chin and the upper and middle facial third asymmetry is still controversial. López Buitrago et al. reported lateral deviation of chin is associated with upper and middle facial third asymmetry, while Kwon et al. reported lateral deviation of chin is not closely related with upper and middle facial third asymmetry [7, 12].The purpose of this study was to evaluate the correlation of upper and middle facial third asymmetry with lateral deviation of chin using 3-dimensional computed tomography (3-D CT).

\section{Methods}

\section{Patients}

The study was conducted on patients who underwent orthognathic surgery at the Department of Oral and Maxillofacial surgery, Gangnam Severance Hospital, Seoul, Korea, between January 2016 and August 2017. Patients with (1) history of trauma to the jaw and (2) congenital deformities, such as cleft lip and/or palate, were excluded from this study. Finally, 40 patients (18 males and 22 females; mean age, 25.50 years [range, 19 to 42]) were included this retrospective study. This study was approved by Gangnam Severance Hospital Institutional Review Board (Approval No. 3-2019-0119)

\section{Image acquisition and analysis}

A spiral scanner was used for 3-D CT scans advised before orthognathic surgery for pre-surgical evaluation. (SOMATOM sensation 64; Siemens, Erlangen, Germany). During the process of CT scan, the patient's teeth were maintained in centric occlusion, and the scan was obtained with following settings: gantry angle of $0^{\circ}, 1024 \times 1024$ matrix, $120 \mathrm{kV}$, $90 \mathrm{~mA}, 1.0 \mathrm{~mm}$ slice thickness, and $0.5 \mathrm{sec}$ gantry rotation time. The CT analysis software was used to reconstruct the digital imaging and communication in medicine (DICOM) images into 3-D images (Mimics version 23.0; Materalise Dental, Leuven, Belgium).

\section{3-dimensional reference plane and craniofacial landmarks} Landmarks were assigned on the reconstructed 3-D image, and their locations were verified on the axial, midsagittal, and coronal slices. The landmarks and measurements of the craniofacial structures to be performed were selected with reference to previous studies [12, 13]. The various landmarks studies are summarized in Figs. 1, 2 and Table 1.

To determine the standard orientation, 3-D reference planes were initially located. The axial plane (AxP) was defined as a plane including the porion (Po) on both sides and the left orbitale (OrL). The midsagittal plane (MSP) was defined as a plane perpendicular to the AxP, including the crista galli $(\mathrm{Cr})$ and the midpoint of the anterior clinoid process $(\mathrm{Cl})$. The coronal plane $(\mathrm{CoP})$ was defined as a plane perpendicular to the $\mathrm{AxP}$ and the MSP passing through opisthion (Op).

\section{Craniofacial measurements}

The craniofacial measurements performed in the study are summarized in Table 2. Distance between the menton (Me) and MSP was defined as $\mathrm{dMe}$, for convenience of comparison; (+) indicated right side deviation of menton. In the mandible, distance from the condylar superius (Con) to Me, distance from gonion (Go) to Me, and the distance from Con to Go were defined as mandibular length (dML), mandibular body length (dMBL), and ramal height $(\mathrm{dRH})$, respectively. Distance from glenoid
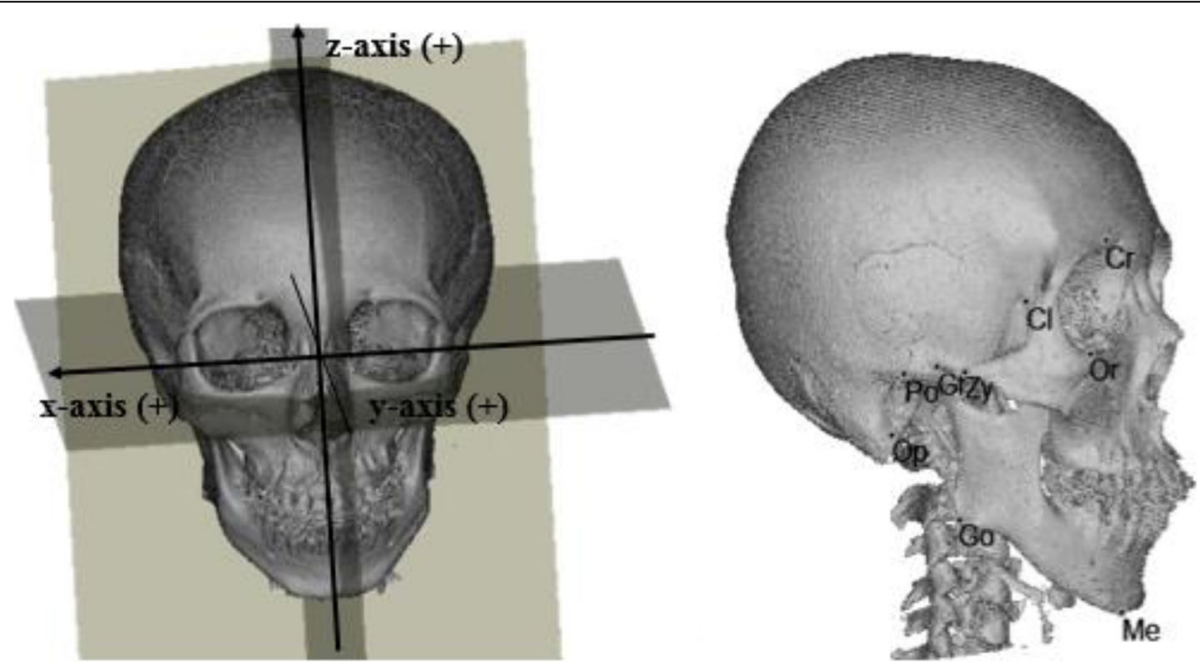

Fig. 1 Three-dimensional reference planes and craniofacial landmarks. Cr, crista galli; Cl, clinoid process; Op, opisthion; Po, porion; Me, menton; Go, gonion; Gf, glenoid fossa; Or, orbitale; Zy, zygonion 


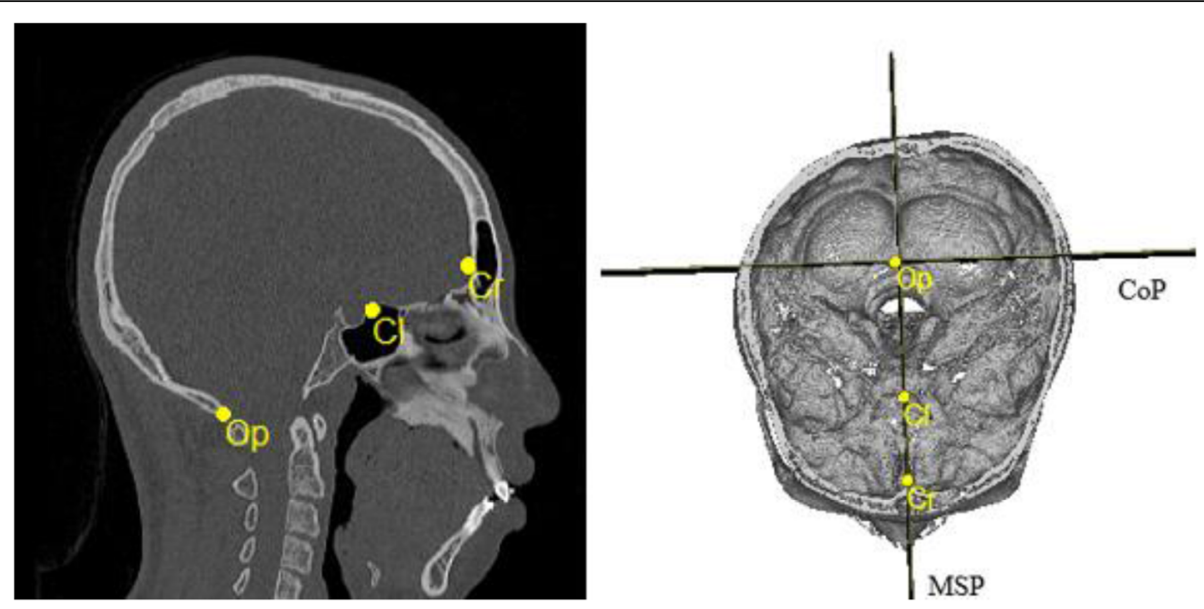

Fig. 2 Computed tomography (CT) scans of craniofacial landmarks. Cr, crista galli; Cl, clinoid process; Op, opisthion; MSP, midsagittal plane; CoP, coronal plane

fossa (Gf) to MSP, CoP and AxP was defined as dGfx, dGfy, and dGfz, respectively. Distance from orbitale (Or) and zygonion $(\mathrm{Zy})$ to each plane was defined as the same way.

Differences between the measurements of distances in the paired craniofacial structures are given in Table 3. $(\mathrm{R}-\mathrm{L})$ was the mean difference between the measurements of distances in the paired craniofacial structures. (R-L) from MSP, CoP, and AxP was defined as $x(R-L)$, $y(R-L)$, and $z(R-L)$. A positive value of $x(R-L)$ indicates that the right craniofacial structure is more lateral than the left craniofacial structure from MSP, positive value of $y(R-L)$ indicates that the right craniofacial structure is more anterior than the left craniofacial structure from $\mathrm{CoP}$, and positive value of $\mathrm{z}(\mathrm{R}-\mathrm{L})$ indicates that the right craniofacial structure is more superior than the left craniofacial structure from AxP. The relationship between the measurements of distances in the paired craniofacial structures and chin deviation was studied.

\section{Statistical analysis}

To avoid inter-observer errors in measurements, all the measurements were performed by a single observer. The Pearson's correlation analysis was performed to evaluate the correlation between chin deviation and difference between the measurements of distances in paired craniofacial structures. Statistical analysis was performed at a significance level of 5\% with SPSS version 25.0 (IBM Corp, Armonk, NY, USA).

Table 1 Description of craniofacial landmarks and reference planes

\begin{tabular}{ll}
\hline Point & Definition \\
\hline Cr (crista galli) & The superior-most edge of the crista galli \\
Cl (clinoid process) & Midpoint between the anterior clinoid processes \\
Con (condylar superius) & The superior-most point of the condylar head; ConR: right, ConL: left \\
Op (opisthion) & Midpoint of the posterior arch of foramen magnum \\
Po (porion) & The superior-most point of the external auditory meatus; PoR: right, PoL: left \\
Me (menton) & The inferior-most point on the symphysis of mandible \\
Go (gonion) & The apex of the mandibular angle; GoR: right, GoL: left \\
Gf (glenoid fossa) & The antero-superior-most point of the glenoid fossa; GfR: right, GfL: left \\
Or (orbitale) & The inferior-most point of the infraorbital rim; OrR: right, OrL: left \\
Zy (zygonion) & The lateral-most point of the zygomatic arch; ZyR: right, ZyL: left \\
Axial plane (AxP) & A plane passing through PoR, PoL, and OrL \\
Midsagittal plane (MSP) & A plane perpendicular to the axial plane including Cr and Cl \\
Coronal plane (CoP) & A plane perpendicular to the axial plane and sagittal planes passing through Op \\
\hline
\end{tabular}


Table 2 Description of craniofacial measurements of distances

\begin{tabular}{|c|c|c|}
\hline & Definition & Description \\
\hline $\mathrm{dMe}$ & Distance from Me to midsagittal plane & $(+)$ indicates right side deviation of menton \\
\hline $\mathrm{dML}$ & Distance from Con to Me & dMLR: right, dMLL: left \\
\hline $\mathrm{dMBL}$ & Distance from Go to Me & dMBLR: right, dMBLL:left \\
\hline $\mathrm{dRH}$ & Distance from Con to Go & dRHR: right, dRHL: left \\
\hline$d G f x$ & Distance from Gf to midsagittal plane & dGfxR: right, dGfxL: left \\
\hline dOrx & Distance from Or to midsagittal plane & dOrxR: right, dOrxL: left \\
\hline$d Z y x$ & Distance from Zy to midsagittal plane & dZyxR: right, dZyxL: left \\
\hline dGfy & Distance from Gf to coronal plane & dGfyR: right, dGfyL: left \\
\hline dOry & Distance from Or to coronal plane & dOryR: right, dOryL: left \\
\hline dZyy & Distance from Zy to coronal plane & dZyyR: right, dZyyL: left \\
\hline$d G f z$ & Distance from Gf to axial plane & dGfzR: right, dGfzL: left \\
\hline dOrz & Distance from Or to axial plane & dOrzR: right, dOrzL: left \\
\hline$d z y z$ & Distance from Zy to axial plane & dZyzR: right, dZyzL: left \\
\hline
\end{tabular}

*Abbreviations: $d M L$ refers to mandibular length; $d M B L$ refers to mandibular body length; $d R H$ refers to ramal height; Me, menton; Con, condylar superius; Go, gonion; Gf, glenoid fossa; Or, orbitale; $Z y$, zygonion

The intraclass correlation coefficient was used to evaluate intra-observer error by the same observer 1 week apart. In this study, the second set of measurements was used.

\section{Results}

\section{Study subjects}

The characteristics of patients included in the study are summarized in Table 4. A total of 40 patients (18 males and 22 females; mean age, 25.50 years [range, 19 to 42]) were included in this study. In our study, 18 patients
(45\%) showed chin deviation to the right side, and 22 patients (55\%) showed chin deviation to the left side (mean, $-1.82 \mathrm{~mm}$ [range, $-16.44 \mathrm{~mm}$ to $8.44 \mathrm{~mm}$ ), and $(+)$ indicates right side deviation of menton. Of the 40 patients, skeletal class III, class II, and class I malocclusions were evident in 33,3 , and 4 patients, as determined by lateral cephalograms.

The intraclass correlation coefficients of craniofacial distance measurements are shown in Table 5. The intraclass correlation coefficient ranged from 0.91 to 0.99 , which showed that data from one observer were very reliable.

Table 3 Description of difference between the measurements of distances in paired craniofacial structures.

\begin{tabular}{|c|c|c|}
\hline & Definition & Description \\
\hline $\mathrm{dML}(\mathrm{R}-\mathrm{L})$ & $\mathrm{dMLR}-\mathrm{dMLL}$ & \multirow[t]{3}{*}{$(\mathrm{R}-\mathrm{L})$ refers to the difference between the measurements of distances in paired craniofacial structures (right-left) } \\
\hline $\mathrm{dMBL}(\mathrm{R}-\mathrm{L})$ & $\mathrm{dMBLR}-\mathrm{dMBLL}$ & \\
\hline $\mathrm{dRH}(\mathrm{R}-\mathrm{L})$ & $\mathrm{dRHR}-\mathrm{dRHL}$ & \\
\hline$d G f x(R-L)$ & $d G f x R-d G f x L$ & \multirow{3}{*}{$\begin{array}{l}\times(R-L) \text { refers to the difference between the measurements of distances in paired craniofacial structures from MSP, and (+) } \\
\text { indicates that the right craniofacial structure is more lateral than the left craniofacial structure from MSP }\end{array}$} \\
\hline$d Z y x(R-L)$ & $d Z y x R-d Z y x L$ & \\
\hline $\mathrm{dOrx}(\mathrm{R}-\mathrm{L})$ & dOrxR-dOrxL & \\
\hline dGfy(R-L) & dGfyR-dGfyL & \multirow{3}{*}{$\begin{array}{l}y(R-L) \text { refers to the difference between the measurements of distances in paired craniofacial structures from CoP, and }(+) \\
\text { indicates that the right craniofacial structure is more anterior than left craniofacial structure form CoP }\end{array}$} \\
\hline$d Z y y(R-L)$ & $d Z y y R-d Z y y L$ & \\
\hline dOry(R-L) & dOryR-dOryL & \\
\hline$d G f z(R-L)$ & $d G f z R-d G f z L$ & \multirow{3}{*}{$\begin{array}{l}z(R-L) \text { refers to the difference between the measurements of distances in paired craniofacial structures from AxP, and (+) } \\
\text { indicates that the right craniofacial structure is more superior to the left craniofacial structure }\end{array}$} \\
\hline$d z y z(R-L)$ & $d Z y z R-d Z y z L$ & \\
\hline dOrz(R-L) & dOrzR-dOrzL & \\
\hline
\end{tabular}

*Abbreviations: $(R-L)$, difference between measurements of distances in paired craniofacial structures (right-left); $d M L$ refers to mandibular length; $d M B L$ refers to mandibular body length; $d R H$ refers to ramal height; $d G f x$, distance from glenoid fossa to midsagittal plane; $d Z y x$, distance from zygonion to midsagittal plane; $d O r x$, distance from orbitale to midsagittal plane; $d G f y$, distance from glenoid fossa to coronal plane; $d z y y$, distance from zygonion to coronal plane; $d O r y$, distance from orbitale to coronal plane; $d G f z$, distance from glenoid fossa to axial plane; $d Z y z$, distance from zygonion to axial plane; $d O r z$, distance from orbitale to axial plane 
Table 4 Patients characteristics $(N=40)$.

\begin{tabular}{lll}
\hline Characteristics & Categories & $\begin{array}{l}\text { Number } \\
\text { (percent) }\end{array}$ \\
\hline Gender & Male & $18(45 \%)$ \\
Age (years) & Female & $22(55 \%)$ \\
& Mean & 25.50 \\
Chin deviation (direction) & Range & 19 to 42 \\
& Right & $18(45 \%)$ \\
Chin deviation (mm) & Left & $22(55 \%)$ \\
(+) indicates right side deviation & Mean & -1.82 \\
of menton & Range & -16.44 to 8.44 \\
Type of skeletal malocclusion & Skeletal class I & $4(10 \%)$ \\
& malocclusion & \\
& Skeletal class II & $3(8 \%)$ \\
& malocclusion & \\
& $\begin{array}{l}\text { Skeletal class III } \\
\text { malocclusion }\end{array}$ & $33(82 \%)$ \\
\hline
\end{tabular}

The correlation between chin deviation and difference between the measurements of distances in paired lower facial third structures

The correlation between chin deviation and difference between the measurements of distances in paired lower facial third structures is shown in Table 6. In the lower facial thirds, the degree of chin deviation was related to mandibular length and mandibular body length. Mandibular length and mandibular body length are shorter on the deviatedchin side compared to that on the non-deviated side (mandibular length, $r=-0.897$, value $<0.001$; mandibular body length, $r=-0.318, p$ value $=0.045)$. However, no significant relation was observed between the degree of chin deviation and ramal height.

\section{The correlation between chin deviation and difference between the measurements of distances in paired upper and middle facial third structures}

The correlation between chin deviation and difference between the measurements of distances in paired upper and middle facial third structures is shown in Table 7. In the upper and middle facial thirds, the degree of chin deviation was correlated with the vertical asymmetry of glenoid fossa and zygonion. Glenoid fossa and zygonion were superior on the deviated-chin side compared to that on the non-deviated side (glenoid fossa, $r=0.317$, $p$ value $=0.046$; zygonion, $r=0.357, p$ value $=0.024)$. However, no significant relation was observed between the degree of chin deviation and position of orbitale.

\section{Discussion}

The purpose of this study was to evaluate the correlation between upper and middle facial third asymmetry and lateral deviation of chin using 3-D CT.

Traditionally, posteroanterior cephalograms, submentovertex view radiographs, or frontal facial photos have been used for diagnosing facial asymmetry. Certainly, these diagnostic modalities have proven their worth over the years. However, they have limited diagnostic abilities due to problems related to magnification, distortion, and superimposition of craniofacial structures [14-17]. However, 3-D CT reduces errors due to magnification and

Table 5 Intraclass correlation coefficient of craniofacial distance measurements $(N=40)$.

\begin{tabular}{lll}
\hline & Intraclass correlation coefficient (single) & 95\% confidence interval (single) \\
\hline$d M e$ & 0.947 & $0.903-0.972$ \\
$d M L$ & $d M L R: 0.994, d M L L: 0.994$ & $d M L R: 0.988-0.997, d M L L: 0.988-0.997$ \\
$d M B L$ & $d M B L R: 0.987, d M B L L: 0.988$ & $d M B L R: 0.976-0.993, d M B L L: 0.977-0.994$ \\
$d R H$ & $d R H R: 0.993, d R H L: 0.994$ & $d R H R: 0.987-0.996, d R H L: 0.988-0.997$ \\
$d G f x$ & $d G f x R: 0.972, d G f x L: 0.965$ & $d G f x R: 0.948-0.985, d G f x: 0.934-0.981$ \\
$d O r x$ & $d O r x R: 0.971, d O r x L: 0.943$ & $d O r x R: 0.945-0.984, d O r x L: 0.895-0.969$ \\
$d Z y x$ & $d Z y x: 0.983, d Z y x: 0.941$ & $d Z y x R: 0.968-0.991, d Z y x L: 0.891-0.968$ \\
$d G f y$ & $d G f y R: 0.973, d G f y L: 0.914$ & $d G f y R: 0.949-0.986, d G f y L: 0.844-0.954$ \\
$d O r y$ & $d O r y R: 0.982, d O r y L: 0.989$ & $d O r y R: 0.967-0.991, d O r y L: 0.980-0.994$ \\
$d Z y y$ & $d Z y y: 0.994, d Z y y L: 0.987$ & $d Z y y: 0.989-0.997, d Z y y L: 0.976-0.993$ \\
$d G f z$ & $d G f z R: 0.982, d G f z: 0.910$ & $d G f z R: 0.966-0.990, d G f z:: 0.836-0.911$ \\
$d O r z$ & $d O r z R: 0.994$ & $d O r z R: 0.988-0.997$
\end{tabular}

*Abbreviations: $d M e$, distance from Me to midsagittal plane; $d M L$, mandibular length; $d M B L$, mandibular body length; $d R H$, ramal height; $d G f x$, distance from glenoid fossa to midsagittal plane; $d Z y x$, distance from zygonion to midsagittal plane; $d O r x$, distance from orbitale to midsagittal plane; $d G f y$, distance from glenoid fossa to coronal plane; $d Z y y$, distance from zygonion to coronal plane; $d O r y$, distance from orbitale to coronal plane; $d G f z$, distance from glenoid fossa to axial plane; $d z y z$, distance from zygonion to axial plane; $d O r z$, distance from orbitale to axial plane 
Table 6 The correlation between chin deviation and difference between the measurements of distances in paired lower facial third structures $(N=40)$.

\begin{tabular}{lll}
\hline & \multicolumn{1}{l}{$\mathrm{dMe}$} & \\
\cline { 2 - 3 } & $R$ & $p$ value \\
\hline $\mathrm{dML}(\mathrm{R}-\mathrm{L})$ & -0.897 & $<0.001$ \\
$\mathrm{dMBL}(\mathrm{R}-\mathrm{L})$ & -0.318 & 0.045 \\
$\mathrm{dRH}(\mathrm{R}-\mathrm{L})$ & -0.123 & 0.449 \\
\hline
\end{tabular}

*Abbreviations: (R-L), difference between the measurements of distances in paired craniofacial structures (right-left); $d M L$ refers to mandibular length; $d M B L$ refers to mandibular body length; $d R H$ refers to ramal height

distortion and allows the quantitative measurements of craniofacial structures [18-20].

Currently, the external auditory meatus is regarded as a reliable reference for the analysis of craniofacial characteristics because of its stable shape [21]. Previous 3-D studies use the Frankfort's horizontal plane as the reference axial plane [22-24]. For these reasons, in this study, the Frankfort's horizontal plane passing through bilateral porion and left orbitale was used as the axial plane. Then, a plane perpendicular to the axial plane passing through the crita galli $(\mathrm{Cr})$ and the midpoint between the anterior clinoid processes $(\mathrm{Cl})$ was defined as midsagittal plane [12]. A plane perpendicular to axial and midsagittal plane with passing through opisthion $(\mathrm{Op})$ was defined as a coronal plane based on the study of Kwon et al .[12].

The glenoid fossa is a depression in the temporal bone that articulates with the mandible to form the

Table 7 The correlation between chin deviation and difference between the measurements of distances in paired upper and middle facial third structures $(N=40)$.

\begin{tabular}{lll}
\hline & \multicolumn{2}{l}{$\mathrm{dMe}$} \\
\cline { 2 - 3 }$R$ & 0.017 & $p$ value \\
\hline dGfx (R-L) & 0.918 \\
dZyx (R-L) & 0.310 & 0.051 \\
dOrx (R-L) & 0.120 & 0.460 \\
dGfy (R-L) & 0.018 & 0.914 \\
dZyy (R-L) & 0.099 & 0.545 \\
dOry (R-L) & 0.033 & 0.838 \\
dGfz (R-L) & 0.317 & 0.046 \\
dZyz (R-L) & 0.357 & 0.024 \\
dOrz (R-L) & 0.189 & 0.242 \\
\hline
\end{tabular}

*Abbreviations: $(R-L)$, difference between the measurements of distances in paired craniofacial structures (right-left); $d G f x$, distance from glenoid fossa to midsagittal plane; $d Z y x$, distance from zygonion to midsagittal plane; $d O r x$, distance from orbitale to midsagittal plane; $d G f y$, distance from glenoid fossa to coronal plane; $d Z y y$, distance from zygonion to coronal plane; $d O r y$, distance from orbitale to coronal plane; $d G f z$, distance from glenoid fossa to axial plane; $d z y z$, distance from zygonion to axial plane; $d O r z$, distance from orbitale to axial plane temporomandibular joint [25]. Positional changes in the glenoid fossa during growth can lead to facial asymmetry and malocclusion [7]. The location of the orbit and zygomatic bone plays an important role in facial symmetry and esthetics [26, 27]. For these reasons, the glenoid fossa, orbitale, and zygomatic arch were analyzed in this study. Mandibular length, mandibular body length, and ramal height were also analyzed to evaluate lower facial third asymmetry.

In the lower facial thirds, chin deviation is correlated with mandibular length and mandibular body length asymmetry, coincident with the findings of previous studies [12, 21, 28]. Moreover, in our study, chin deviation was also correlated with the upper and middle facial third asymmetry, especially vertical asymmetry of the glenoid fossa and zygomatic arch, coincident with the findings of another stud y[7]. In a previous study, asymmetry of the glenoid cavity, a type of upper and middle facial third asymmetry, was reported [7]. The asymmetry of glenoid cavity is often caused by the defects in generation, proliferation, migration, and differentiation of cranial neural crest cells [8] or craniofacial structure modeling from the cerebrum [9-11]. As a result, the glenoid cavity is located superiorly where developmental defects occurred (affected side) [7]. Similarly, supraorbital arch, zygomatic bone, and external auditory meatus are also located superiorly on affected side (orbiculo-zygomatic-meatal and articular asymmetry) [7]. Finally, the asymmetry of glenoid cavity functionally affects condylar position, causing lateral deviation of chin to the affected side [7].

This study showed that lateral deviation of chin is correlated with upper and middle facial third asymmetry as well as lower facial third asymmetry, especially vertical asymmetry of the glenoid fossa and zygomatic arch. Correction of chin deviation by mandibular surgery alone will not correct the asymmetry of the upper and middle facial thirds.

A limitation of this study is that a small number of craniofacial landmarks were analyzed for the correlation with the lateral deviation of chin, and further studies incorporating more number of craniofacial landmarks should be conducted for a deeper understanding of the correlation between the lateral deviation of chin and craniofacial landmarks.

To be best of our knowledge, this is the first study to evaluate craniofacial characteristics associated with the lateral deviation of chin using 3-dimensional imaging modalities. Considering the high prevalence and the impact of facial asymmetry on patient's treatment outcome, this study is very relevant in the present scenario. Knowledge about the fact that facial symmetry is influenced by the upper and middle thirds of face will help clinicians around the world in proper treatment planning and hence, in providing better treatment to such patients. 


\section{Conclusions}

Lateral deviation of chin is correlated with upper and middle facial third asymmetry as well as lower facial third asymmetry. Correction of chin deviation by mandibular surgery alone will not correct the asymmetry of the upper and middle facial thirds. As a result, treatment planning in patients with chin deviation should involve a careful evaluation of the asymmetry of the upper and middle facial thirds to ensure complete patient satisfaction.

\begin{abstract}
Abbreviations
(R-L): Difference between the measurements of distances in paired craniofacial structures (right-left); 3-D CT: 3-dimensional computed tomography; AxP: Axial plane; Cl: Clinoid process; Con: Condylar superius; ConL: Left condylar superius; ConR: Right condylar superius; CoP: Coronal plane; Cr: Crista galli; dGfx: Distance from Gf to midsagittal plane; dGfx(RL): dGfxR-dGfxL; dGfxL: Left dGfx; dGfxR: Right dGfx; dGfy: Distance from Gf to coronal plane; dGfy(R-L): dGfyR-dGfyL; dGfyL: Left dGfy; dGfyR: Right dGfy; dGfz: Distance from Gf to axial plane; dGfz(R-L): dGfzR-dGfzL; dGfzL: Left dGfz; dGfzR: Right dGfz; DICOM: Digital imaging and communication in medicine; dMBL: Mandibular body length, distance from Go to Me; $\mathrm{dMBL}(\mathrm{R}$ L): dMBLR-dMBLL; dMe: Distance from Me to midsagittal plane; dML: Mandibular length, distance from Con to Me; dML(R-L): dMLR-dMLL; dOrx: Distance from Or to midsagittal plane; dOrx(R-L): dOrxR-dOrxL; dOrxL: Left dOrx; dOrxR: Right dOrx; dOry: Distance from Or to coronal plane; dOry(R-L): dOryR-dOryL; dOryL: Left dOry; dOryR: Right dOry; dOrz: Distance from Or to axial plane; dOrz(R-L): dOrzR-dOrzL; dOrzL: Left dOrz; dOrzR: Right dOrz; dRH: Ramal height, distance from Con to Go; dRH(R-L): dRHR-dRHL; dZyx: Distance from Zy to midsagittal plane; dZyx(R-L): dZyxR-dZyxL; dZyxL: Left dZyx; dZyxR: Right dZyx; dZyy: Distance from Zy to coronal plane; $d Z y y(R-L)$ : dZyyR-dZyyL; dZyyL: Left dZyy; dZyyR: Right dZyy; dZyz(RL): dZyzR-dZyzL; dZyz: Distance from Zy to axial plane; dZyzL: Left dZyz; dZyzR: Right dZyz; Gf: Glenoid fossa; GfL: Left glenoid fossa; GfR: Right glenoid fossa; Go: Gonion; GoL: Left gonion; GoR: Right gonion; Me: Renton; MSP: Midsagittal plane; Op: Opithion; Or: Orbitale; OrL: Left orbitale; OrR: Right orbitale; Po: Porion; PoL: Left porion; PoR: Right porion; x(R$\mathrm{L})$ : Difference between the measurements of distances in paired craniofacial structures from MSP; $y(R-L)$ : Difference between the measurements of distances in paired craniofacial structures from CoP; $z(R-L)$ : Difference between the measurements of distances in paired craniofacial structures from AxP; Zy: Zygonion; ZyL: Left zygonion; ZyR: Right zygonion
\end{abstract}

\section{Acknowledgements}

We would like to thank Editage (www.editage.co.kr) for English language editing.

\section{Authors' contributions}

HWC and BK were responsible for collecting and analyzing the data. JYK was responsible for statistical analysis and translation of the data. KHP and JKH contributed to the direction and design of the research and contributed to the review of the paper. All the authors have read the final version of the paper and have agreed to its publication.

\section{Funding}

This research received no specific grant from any funding agency in the public, commercial, or not-for profit sectors.

\section{Availability of data and materials}

The data sets used and/or analyzed during the current study are available from the corresponding author on reasonable request.

\section{Ethics approval and consent to participate}

This study was approved by Gangnam Severance Hospital Institutional Review Board (Approval No. 3-2019-0119).

\section{Consent for publication}

Not applicable.

\section{Competing interests}

The authors declare that they have no competing interests.

Received: 5 September 2019 Accepted: 20 November 2019

Published online: 06 December 2019

\section{References}

1. Haraguchi S, Takada K, Yasuda Y (2002) Facial asymmetry in subjects with skeletal class III deformity. Angle Orthod 72:28-35

2. Samman N, Tong AC, Cheung DL, Tideman H (1992) Analysis of 300 dentofacial deformities in Hong Kong. Int J Adult Orthodon Orthognath Surg 7:181-185

3. Severt TR, Proffit WR (1997) The prevalence of facial asymmetry in the dentofacial deformities population at the University of North Carolina. Int J Adult Orthodon Orthognath Surg 12:171-176

4. Burstone CJ (1998) Diagnosis and treatment planning of patients with asymmetries. Semin Orthod 4:153-164

5. Obwegeser HL, Makek MS (1986) Hemimandibular hyperplasia-hemimandibular elongation. J Maxillofac Surg 14:183-208

6. Poikela A, Kantomaa T, Pirttiniemi P (1997) Craniofacial growth after a period of unilateral masticatory function in young rabbits. Eur J Oral Sci 105: 331-337

7. López Buitrago DF, Ruiz Botero J (2017) Asymmetry of glenoid fossa as differential diagnosis for hemimandibular elongation. Revista Mexicana de Ortodoncia 5:e217-ee26

8. Minoux M, Rijli FM (2010) Molecular mechanisms of cranial neural crest cell migration and patterning in craniofacial development. Development 137: 2605-2621

9. de Cráneo AdIB, el Crecimiento D (2011) Asymmetry of human skull base during growth. Int J Morphol 29:1028-1032.

10. Basili C, Costa H, Sasaguri K, Akimoto S, Slavicek R, Sato S (2009) Comparison of the position of the mandibular fossa using 3D CBCT in different skeletal frames in human caucasic skulls. Int J Stomatol Occlusion Med 2:179-190

11. Slavicek R (2001) The dynamic functional anatomy of craniofacial complex and its relation to the articulation of the dentitions. Das Kauorgan Funktione und Dysfunktionen:482-514

12. Kwon TG, Park HS, Ryoo HM, Lee SH (2006) A comparison of craniofacial morphology in patients with and without facial asymmetry-a threedimensional analysis with computed tomography. Int J Oral Maxillofac Surg 35:43-48

13. You KH, Lee KJ, Lee SH, Baik HS (2010) Three-dimensional computed tomography analysis of mandibular morphology in patients with facial asymmetry and mandibular prognathism. Am J Orthod Dentofacial Orthop 138:540.e1-540.e8 discussion -1

14. Ahlqvist J, Eliasson S, Welander U (1983) The cephalometric projection. part II. principles of image distortion in cephalography. Dentomaxillofac Radiol 12:101-108

15. Bergersen EO (1980) Enlargement and distortion in cephalometric radiography: compensation tables for linear measurements. Angle Orthod 50:230-244

16. Gravely JF, Benzies PM (1974) The clinical significance of tracing error in cephalometry. Br J Orthod 1:95-101

17. Savara BS, Tracy WE, Miller PA (1966) Analysis of errors in cephalometric measurements of three-dimensional distances on the mandible. Arch Oral Biol 11:209-217

18. Fuhrmann RA, Schnappauf A, Diedrich PR (1995) Three-dimensional imaging of craniomaxillofacial structures with a standard personal computer Dentomaxillofac Radiol 24:260-263

19. Katsumata A, Fujishita M, Maeda M, Ariji Y, Ariji E, Langlais RP (2005) 3D-CT evaluation of facial asymmetry. Oral Surg Oral Med Oral Pathol Oral Radiol Endod 99:212-220

20. Xia J, Ip HH, Samman N, Wang D, Kot CS, Yeung RW et al (2000) Computerassisted three-dimensional surgical planning and simulation: 3D virtual osteotomy. Int J Oral Maxillofac Surg 29:11-17

21. Kim YH, Sato K, Mitani H, Shimizu Y, Kikuchi M (2003) Asymmetry of the sphenoid bone and its suitability as a reference for analyzing craniofacial asymmetry. Am J Orthod Dentofacial Orthop 124:656-662

22. Captier G, Leboucq N, Bigorre M, Canovas F, Bonnel F, Bonnafe A et al (2003) Plagiocephaly: morphometry of skull base asymmetry. Surg Radiol Anat 25:226-233 
23. Lo LJ, Marsh JL, Pilgram TK, Vannier MW (1996) Plagiocephaly: differential diagnosis based on endocranial morphology. Plast Reconstr Surg 97:282-291

24. Yu CC, Wong FH, Lo LJ, Chen YR (2004) Craniofacial deformity in patients with uncorrected congenital muscular torticollis: an assessment from threedimensional computed tomography imaging. Plast Reconstr Surg 113:24-33

25. Sato S, Takamoto K, Fushima K, Akimoto S, Suzuki Y (1989) A new orthodontic approach to mandibular lateral displacement malocclusionimportance of occlusal plane reconstruction. Dent Jpn 26:81-85

26. Springer IN, Wannicke B, Warnke PH, Zernial O, Wiltfang J, Russo PA et al (2007) Facial attractiveness: visual impact of symmetry increases significantly towards the midline. Ann Plast Surg 59:156-162

27. Khaqani MS, Tavosi F, Gholami M, Eftekharian HR, Khojastepour L (2018) Analysis of facial symmetry after zygomatic bone fracture management. J Oral Maxillofac Surg 76:595-604

28. Baek C, Paeng JY, Lee JS, Hong J (2012) Morphologic evaluation and classification of facial asymmetry using 3-dimensional computed tomography. J Oral Maxillofac Surg 70:1161-1169

\section{Publisher's Note}

Springer Nature remains neutral with regard to jurisdictional claims in published maps and institutional affiliations.

\section{Submit your manuscript to a SpringerOpen ${ }^{\circ}$ journal and benefit from:}

- Convenient online submission

- Rigorous peer review

- Open access: articles freely available online

High visibility within the field

- Retaining the copyright to your article

Submit your next manuscript at $\boldsymbol{\wedge}$ springeropen.com 